PROCEEDINGS OF THE

AMERICAN MATHEMATICAL SOCIETY

Volume 135, Number 4, April 2007, Pages 1169-1173

S 0002-9939(06)08562-5

Article electronically published on November 13, 2006

\title{
DIMENSION DISTORTION OF HYPERBOLICALLY CONVEX MAPS
}

\author{
S. ROHDE
}

(Communicated by Juha M. Heinonen)

\begin{abstract}
In this note, we provide an answer to a question of D. Mejia and Chr. Pommerenke, by constructing a hyperbolically convex subdomain $G$ of the unit disc $\mathbb{D}$ so that the conformal map from $\mathbb{D}$ to $G$ maps a set of dimension 0 on $\partial \mathbb{D}$ to a set of dimension 1 .
\end{abstract}

\section{INTRODUCTION AND STATEMENT OF THE RESULT}

A subdomain $G$ of the unit disc $\mathbb{D}$ in $\mathbb{C}$ is hyperbolically convex if it is convex with respect to the hyperbolic metric of $\mathbb{D}$. These domains are just intersections of hyperbolic halfplanes. Conformal maps from $\mathbb{D}$ onto hyperbolically convex domains have been systematically studied beginning with Ma and Minda (2] and [3]); see the papers [5], 6] and [7] by Mejia and Pommerenke for further references. Hyperbolically convex domains appear naturally in various situations such as the Hayman-Wu problem, where the Brown-Flynns theorem (the boundary of hyperbolically convex domains has length $<\pi^{2}$ ) has been used by Fernández, Heinonen and Martio [1] to obtain the bound $4 \pi^{2}$ for the Hayman-Wu constant (see [9] for the best bound to date and for references).

In 5, Mejia and Pommerenke proved that, for conformal maps $f$ of $\mathbb{D}$ onto hyperbolically convex domains,

$$
\operatorname{dim} f(A)<1
$$

if $A \subset \mathbb{T}$ has $\operatorname{dim} A<1$ and if $f(A)$ is uniformly perfect. They asked (Conjecture 3 in [6]) if the assumption of uniform perfectness can be omitted. We will answer this question in the negative by proving

Theorem 1. There is a hyperbolically convex domain $G \subset \mathbb{D}$ and a set $A \subset \mathbb{T}$ with $\operatorname{dim} A=0$ and $\operatorname{dim} f(A)=1$, where $f$ is the conformal map from $\mathbb{D}$ onto $G$.

Our domain is of Schottky type (meaning that the complement of $\bar{G}$ is a union of disjoint hyperbolic halfplanes; see $[5$ and Theorem 2 therein). The inductive construction of the domain $G$ as a decreasing sequence of hyperbolically convex domains $G_{n}$ is slightly easier to describe in the upper halfplane $\mathbb{H}$ : Fix a sequence $\varepsilon_{1}, \varepsilon_{2}, \ldots$ of small positive numbers and positive integers $n_{1}, n_{2}, \ldots$ (to be determined later). Next, construct a Cantor-like set $S \subset[0,1] \subset \mathbb{R}$ in the following way.

Received by the editors November 9, 2004 and, in revised form, November 16, 2005.

2000 Mathematics Subject Classification. Primary 30C35.

The author was partially supported by NSF Grants DMS-0201435 and DMS-0244408.

(C)2006 American Mathematical Society Reverts to public domain 28 years from publication 


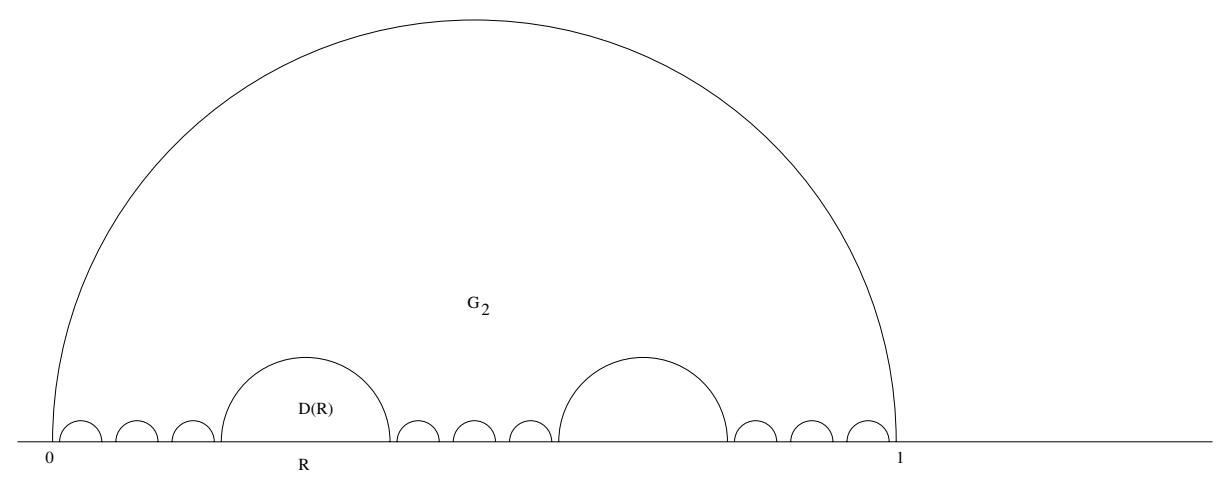

FiguRE 1.1. The second approximation to the hyperbolically convex domain $G$

Set $S_{0}=[0,1]$. Suppose that $S_{k}$ has already been constructed and consists of $N_{k}=n_{1} n_{2} \cdots n_{k}$ intervals $I_{j}^{(k)}$ of size

$$
s_{k}=\left(\varepsilon_{1} \varepsilon_{2} \cdots \varepsilon_{k}\right) /\left(n_{1} n_{2} \cdots n_{k}\right) .
$$

Form $S_{k+1}$ from $S_{k}$ by replacing each of the $I_{j}^{(k)}$ by $n_{k+1}$ equally spaced subintervals $I_{\ell}^{(k+1)}$ of size $s_{k+1}=\left(\varepsilon_{1} \varepsilon_{2} \cdots \varepsilon_{k+1}\right) /\left(n_{1} n_{2} \cdots n_{k+1}\right)$. Set

$$
S=\bigcap_{k} \bigcup_{j}^{(k)}
$$

For instance, the middle-third Cantor set is the special case $n_{k}=2$ and $\varepsilon_{k}=2 / 3$. Denote the "removed" invervals by $R_{\ell}^{(k+1)}, 1 \leq \ell \leq\left(n_{k+1}-1\right) n_{1} \cdots n_{k}$. For an interval $I \subset[0,1]$, let $D(I)$ be the open halfdisc in $\mathbb{H}$ with diameter $I$. Set

$$
G_{k}=D([0,1]) \backslash \bigcup_{1 \leq m \leq k} \bigcup_{j} \overline{D\left(R_{j}^{(m)}\right)}
$$

and

see Figure 1.1.

$$
G=\bigcap G_{k}
$$

Fix a point $z_{0} \in G$. Let $g_{k}$, respectively $g$, denote the conformal map of $G_{k}$, respectively $G$, onto $\mathbb{D}$ normalized by $g_{k}\left(z_{0}\right)=0$ and $g_{k}^{\prime}\left(z_{0}\right)>0$. Because $\partial G$ is a simple curve, $g$ extends continuously to $\bar{G}$. We will show that an appropriate choice of the parameters $\varepsilon_{k}$ and $n_{k}$ yields a set $S$ of dimension 1 such that $g(S)$ has dimension 0, proving the theorem (with $f=g^{-1}$ and $A=g(S)$ ). More precisely, we will prove

Theorem 2. If

$$
\log n_{k}=o\left(\frac{1}{\sqrt{\varepsilon_{k}}}\right) \quad \text { as } \quad k \rightarrow \infty
$$

then

On the other hand, if

$$
\operatorname{dim} g(S)=0
$$

$$
\log \frac{1}{\varepsilon_{k}}=o\left(\log n_{k}\right) \quad \text { as } \quad k \rightarrow \infty,
$$


then

$$
\operatorname{dim} S=1 .
$$

Theorem 1 follows immediately by taking any pair of sequences $\varepsilon_{k}, n_{k}$ that satisfies both conditions, for instance $\varepsilon_{k}=1 / k^{2}$ and $n_{k}=\exp (\sqrt{k})$.

It was realized in [5] that domains of Schottky type are the "critical domains" for the validity of the dimension distortion property: Mejia and Pommerenke proved (Theorem 2 of [ $[$ ) that $\operatorname{dim} A<1$ would imply $\operatorname{dim} f(A)<1$ for all hyperbolically convex $f$ if $\operatorname{dim} A<1$ implies $\operatorname{dim} f(A)<1$ for those hyperbolically convex $f$ that are of Schottky type. Notice that our domain $G$ is of Schottky type, so that the assumption of their Theorem 2 does not hold.

The phenomenon responsible for the strong dimension-distortion is the following: if $\varepsilon_{k}$ is very small and $I$ is one of the $I_{j}^{(k)}$, then near $I$ the domain $G_{k}$ looks like a half-strip, whose sides are the two geodesics ending in the endpoints of $I$. Therefore the harmonic measure of $I$ seen from $z_{0}$ is exponentially smaller than the size $s_{k}$ of I. For our set $S$ to have the properties $\operatorname{dim} S=1$ and $\operatorname{dim} g(S)<1$ we will need $\varepsilon_{k} \rightarrow 0$. Thus $S$ cannot be uniformly perfect, in accordance with Theorem 3 of [5].

\section{Proofs}

The letter $C$ denotes various constants whose value may change even within a line. Recall the definition of $S$ from Section 1 and assume that $\varepsilon_{j}<\frac{1}{2}$ for all $j$. Denote by $\hat{I}_{j}^{(k)}$ the (smaller) arc of $\partial G$ that has the same endpoints as $I_{j}^{(k)}$. Fix $z_{0} \in G$.

Lemma 2.1. For all $j$ and $k$ we have

$$
\omega\left(z_{0}, \hat{I}_{j}^{(k)}\right) \leq K_{1} \exp \left(-K_{2} \sum_{\ell=1}^{k} \frac{1}{\sqrt{\varepsilon_{\ell}}}\right),
$$

where $K_{2}$ is a universal constant and $K_{1}$ depends on $z_{0}$ and $G$ only, but not on $j$ or $k$.

Proof. Fix an interval $I=I_{j_{0}}^{\left(k_{0}\right)}$. Denote by $r_{k}$ the length of the intervals $R_{j}^{(k)}$. Then $\left(n_{k+1}-1\right) r_{k+1}+n_{k+1} s_{k+1}=s_{k}$ so that we have

$$
r_{k+1}=\frac{1-\varepsilon_{k+1}}{n_{k+1}-1} s_{k}>\frac{\varepsilon_{k+1}}{n_{k+1}} s_{k}=s_{k+1} .
$$

It follows that

$$
s_{k+1}<h_{k+1}:=\sqrt{r_{k+1} s_{k+1}}<r_{k+1}<s_{k} .
$$

Let $\Gamma$ denote the family of all curves $\gamma$ in $G$ that join a small (fixed) circle centered at $z_{0}$ with $\hat{I}_{j}^{(k)}$. By conformal invariance of the modulus $M(\Gamma)$ and by Pfluger's theorem (see for instance [8], Chapter 9) we have

$$
\omega\left(z_{0}, \hat{I}_{j}^{(k)}\right) \leq C \exp \left(-\frac{\pi}{M(\Gamma)}\right),
$$

where $C$ depends on $z_{0}$ and the circle but not on $j$ or $k$. Consider the strips $S_{k}=$ $\left\{x+i y: s_{k}<y<h_{k}\right\}$. It follows from (2.1) that they are pairwise disjoint. For $1 \leq k \leq k_{0}$, denote by $Q_{k}$ the component of $G \cap S_{k}$ that separates $I$ from $z_{0}$. (We will assume without much loss of generality that $\Im z_{0}>h_{1}$.) Each $Q_{k}$ is, or is contained in, a topological quadrilateral bounded by two horizontal line segments 
(top and bottom) and two circular arcs (sides) of circles of diameter at least $r_{k}$. The length $\ell_{k}$ of the top is maximal if both sides come from circles of diameter $r_{k}$. Because both circles are separated by an interval $I_{j}^{(k)}$ of size $s_{k}$, it follows that

$$
\ell_{k} \leq s_{k}+2\left(r_{k} / 2-\sqrt{\left(r_{k} / 2\right)^{2}-h_{k}^{2}}\right)
$$

and it thus follows from (2.1) using $\sqrt{1-x} \geq 1-x$ that

$$
\ell_{k}<5 s_{k} .
$$

Denote by $Q_{k}^{\prime}$ the smallest rectangle containing $Q_{k}$ : it has horizontal sides of length $\ell_{k}$ and vertical sides of length $h_{k}-s_{k}$. Every curve $\gamma \in \Gamma$ has to pass through each of the $Q_{k}^{\prime}\left(1 \leq k \leq k_{0}\right)$ and therefore (e.g. [8], Proposition 9.3)

$$
\frac{1}{M(\Gamma)} \geq \sum_{k=1}^{k_{0}} \frac{1}{M\left(Q_{k}^{\prime}\right)}=\sum_{k=1}^{k_{0}} \frac{h_{k}-s_{k}}{\ell_{k}} \geq \sum_{k=1}^{k_{0}} \frac{h_{k}}{5 s_{k}} \geq \frac{1}{5 \sqrt{2}} \sum_{k=1}^{k_{0}} \frac{1}{\sqrt{\varepsilon_{k}}},
$$

where the last inequality follows using $r_{k} / s_{k}=\left(n_{k} / \varepsilon_{k}\right)\left(1-\varepsilon_{k}\right) /\left(n_{k}-1\right)$ and $s_{k}<$ $1 / 2$. The lemma follows from Pfluger's theorem, with $K_{2}=\pi /(5 \sqrt{2})$.

Proof of Theorem 2. For every $k$, we have

$$
g(S) \subset \bigcup_{j} g\left(\hat{I}_{j}^{(k)}\right)
$$

By Lemma 2.1, the arc $g\left(\hat{I}_{j}^{(k)}\right)$ has length

$$
\ell_{j, k} \leq 2 \pi K_{1} \exp \left(-K_{2} \sum_{\ell=1}^{k} \frac{1}{\sqrt{\varepsilon_{\ell}}}\right) .
$$

For every $\alpha>0$,

$$
\sum_{j} \ell_{j, k}^{\alpha} \leq C n_{1} n_{2} \cdots n_{k} \exp \left(-K_{2} \alpha \sum_{\ell=1}^{k} \frac{1}{\sqrt{\varepsilon_{\ell}}}\right)=C \prod_{\ell=1}^{k}\left(n_{\ell} \exp \left(-K_{2} \alpha \frac{1}{\sqrt{\varepsilon_{\ell}}}\right)\right)
$$

and the assumption $\log n_{j}=o\left(\frac{1}{\sqrt{\varepsilon_{j}}}\right)$ implies that the factors go to zero as $j \rightarrow \infty$. Thus $\operatorname{dim} g(S) \leq \alpha$.

To prove that $\operatorname{dim} S=1$ if $\log \left(1 / \varepsilon_{j}\right)=o\left(\log n_{j}\right)$, fix $\alpha<1$ and consider the measure $\mu$ on $S$ defined by

$$
\mu\left(I_{j}^{(k)}\right)=\frac{1}{n_{1} n_{2} \cdots n_{k}}
$$

for all $j$ and $k$. The theorem follows at once from the "easy direction" of Frostman's Lemma ([8], Exercise 10.2.5, or [4, Theorem 8.8) if we can prove that, for every interval $I$, we have

$$
\mu(I) \leq C_{\alpha}|I|^{\alpha} .
$$

To this end, let $k$ be such that $s_{k+1} \leq|I|<s_{k}$. Because $r_{k}>s_{k}$, there is only one index $j$ such that $I_{j}^{(k)} \cap I \neq \emptyset$. Let $t=|I| / s_{k}<1$. Then $I$ can intersect at most $t n_{k+1}+2$ of the $n_{k+1}$ subintervals $I_{\ell}^{(k+1)}$ of $I_{j}^{(k)}$. Therefore

$$
\mu(I) \leq\left(t n_{k+1}+2\right) \frac{1}{n_{1} n_{2} \cdots n_{k+1}} .
$$


By assumption,

for $p$ large enough, so that

$$
\log \frac{1}{\varepsilon_{p}} \leq \frac{1-\alpha}{\alpha} \log n_{p}
$$

$$
\frac{1}{n_{1} n_{2} \cdots n_{k}} \leq C\left(\frac{\varepsilon_{1} \varepsilon_{2} \cdots \varepsilon_{k}}{n_{1} n_{2} \cdots n_{k}}\right)^{\alpha}=C s_{k}^{\alpha} .
$$

Now (2.3) implies that

$$
\mu(I) \leq C\left(t s_{k}^{\alpha}+2 s_{k+1}^{\alpha}\right) \leq C\left(t^{1-\alpha}|I|^{\alpha}+2|I|^{\alpha}\right)
$$

and (2.2) is proved.

\section{ACKNOWLEDGEMENT}

I would like to thank the referee for his suggestions, resulting in improved readability of this paper.

\section{REFERENCES}

[1] J. L. Fernández, J. Heinonen, O. Martio Quasilines and conformal mappings, J. Analyse Math. 52 (1989), 117-132. MR0981499 (90a:30017)

[2] W. C. Ma, D. Minda, Hyperbolically convex functions, Ann. Polon. Math. 60 (1994), no. 1, 81-100. MR.1295110 (95k:30037)

[3] W. C. Ma, D. Minda, Hyperbolically convex functions II, Ann. Polon. Math. 71 (1999), no. 3, 273-285. MR $1704303(2000 \mathrm{j}: 30020)$

[4] P. Mattila, Geometry of sets and measures in Euclidean spaces. Fractals and rectifiability, Cambridge University Press (1995). MR.1333890 (96h:28006)

[5] D. Mejia, Chr. Pommerenke, Hyperbolically convex functions, dimension and capacity, Complex Var. Theory Appl. 47 (2002), no. 9, 803-814. MR1925176 (2003f:30017)

[6] D. Mejia, Chr. Pommerenke, Hyperbolically convex functions, Analysis and applicationsISAAC 2001 (Berlin), 89-95, Int. Soc. Anal. Appl. Comput., 10, Kluwer Acad. Publ., Dordrecht, 2003. MR2022741 (2004k:30108)

[7] D. Mejia, Chr. Pommerenke, On the derivative of hyperbolically convex functions, Ann. Acad. Sci. Fenn. Math. 27 (2002), no. 1, 47-56. MR.1884348 (2003b:30016)

[8] Chr. Pommerenke, Boundary behaviour of conformal maps, Springer (1992). MR 1217706 (95b:30008)

[9] S. Rohde, On the theorem of Hayman and Wu, Proc. Amer. Math. Soc. 130 (2002), no. 2, 387-394. MR 1862117 (2002i:30010)

Department of Mathematics, University of Washington, Seattle, Washington 98195 